\title{
Propagation : a New Operation in a Framework for Abstract Interpretation of Logic Programs
}

\author{
Maurice Bruynooghe and Gerda Janssens \\ Department of Computer Science, Katholieke Universiteit Leuven \\ Celestijnenlaan 200A, B-3001 Heverlee, Belgium \\ e-mail: \{maurice,gerda\}@cs.kuleuven.ac.be
}

\begin{abstract}
Most frameworks for abstract interpretation of logic programs provide abstract operations which safely approximate their concrete counterpart. That means, given a concrete state $S$ described by an abstract state $A S$ and a concrete operation $O$ producing $O(S)$, the corresponding abstract operation $A O$ produces $A O(A S)$ which must describe $O(S)$. We sketch a framework which relaxes this condition and requires that the safe approximation condition is only reached after a propagation step which reexecutes - at the abstract level - all operations leading to $O(S)$ until a stable abstract state is reached. We illustrate the novel framework with a mode analysis which, notwithstanding a very simple abstract domain, reaches on several examples similar precision as mode analysis systems using much complexer abstract domains.
\end{abstract}

\section{Introduction}

Almost all frameworks for abstract interpretation of logic programs apply what one could call a principle of locality. The global state of the computation is distributed over many local environments, one for each clause being executed. In the framework of [1], a local environment keeps track of the state of the variables of the clause it annotates. A correct approximation of an operation which is part of a clause, e.g. unification of a variable $X_{i}$ with a constant, correctly describes the new state of the local variables after execution of the concrete operation, e.g. the variable $X_{i}$ becomes ground and all other local variables $X_{j}$ which possibly share their value with $X_{i}$ cannot retain a mode free. To allow such a safe approximation, the abstract state must be sufficiently detailed. In case one includes a mode such as free which is not closed under substitution (instantiating the variable makes the mode incorrect), then sufficiently detailed information about possible sharing must be included in the abstract state, otherwise all modes free should be turned into any as soon as a single variable is involved in a unification. Notice that the unification between $X_{i}$ and the constant can also affect non-local variables e.g. a variable $Y_{j}$ in the caller's environment of the current clause. That environment no longer reflects the current state of the variable $Y_{j}$. However, there is no interest in the current state of non-local variables. It is the role of procedure exit to update the state of the caller's environment at the point where its state becomes again relevant. 
The same observations remain valid when considering abstract interpretation of constraint logic programs. [9] extend the abstract state with a constraint component listing the concrete constraints between local variables. Although the effect of bindings between non-local variables on the values of local variables is rather easily captured in a possible sharing component between local variables, it is not that easy for numeric constraints. A binding to a local variable can propagate through a chain of non-local constraints and finally bind another local variable. [9] take a very rough approach : variables involved in a non-local constraint are always in a state which is instantiation closed. So one can ignore non-local constraints without compromising the safety of the analysis. In the current paper we develop a different schema and consider a distributed computation where one aims at correctly approximating the global state corresponding to the current program point. When approximating the effect of an operation, say unification of $X_{i}$ with a constant, the change to the local state, i.e. that $X_{i}$ becomes ground, is propagated to the neighbouring environments and the computations already done in that environment are reexecuted. Eventual updates are in turn propagated. For example, assume that $X_{i}$ occurs in the head and that the corresponding variable in the caller's environment is $Y_{i}$. That variable becomes also ground. Suppose a part of the executed computation in the caller's environment was unifying $Y_{i}$ with $Y_{j}$, that computation is reexecuted and $Y_{j}$ becomes also ground. These changes in the state of $Y_{i}$ and $Y_{j}$ are in turn propagated. Eventually a stable state is reached where every variable in every environment safely approximates the concrete value in the program point of the concrete execution. To a certain extent, the idea is a generalisation of the repeat-previous-call strategy mentioned in [1]. Due to the transparency of logic programs, one can "repeat" the execution of an already executed part to obtain a better approximation of the state. For some applications (e.g. [4]) it is necessary to make a distinction between the first execution and the reexecutions for precision reasons.

Apparently, we have a more complex algorithm for abstract interpretation. So, what is gained ? The advantage is that one can use simpler abstract domains and simpler abstract operations, which, when only applied locally, would yield incorrect results. The global propagation mechanism turns the result in a safe approximation. For example, one can perform mode analysis with a very simple abstract domain not including any information about sharing and obtain results which appear as precise as with the complex PROP domain of [10] or the EXP domain of [3].

Finally, notice that it is unlikely that the same effect can be achieved by extending the abstract domain with a "global store" containing all operations already executed. Firstly, there is the problem that a procedure can be defined by different clauses, each performing different operations, on exiting from such a procedure, one would have to keep alternative states for the global store. Secondly, recursion causes an unlimited growth of the global store. 


\section{Sketch of the Framework}

Our framework is an extension of the framework of [1] for logic programs which constructs an abstract AND-OR graph adorned with abstract substitutions. The construction of the graph involves three application specific operations : procedure entry, procedure exit and abstract interpretation of built-ins. Plugging in these three operations which satisfy the correctness conditions stated in the framework guarantees that the final graph describes all concrete AND trees (and hence all derivations) that can occur during execution of the intended class of queries.

We assume programs are in so called normal form i.e. arguments of predicates occurring in the heads and bodies of clauses are distinct variables. This simplifies considerably the procedure entry and procedure exit operations as all effects of unifications are concentrated in built-ins of the form $X_{i}=X_{j}$ and $X_{i}=f\left(X_{j_{1}}, \ldots X_{j_{n}}\right)(n \geq 0)$. We first discuss abstract interpretation of builtins. In [1] a built-in has an abstract call substitution $\beta_{\text {in }}$ which is assumed to be correct. It means that any concrete computation which reaches the program point where the built-in has to be performed has an accumulated substitution whose projection upon the variables of the clause the built-in is part of (upon the local environment of the clause), say $\tau_{i n}$, is described by $\beta_{i n}$, i.e. $\tau_{i n} \in \gamma\left(\beta_{i n}\right)$ with $\gamma$ the concretisation function. Executing the built-in under substitution $\tau_{i n}$ yields a new substitution $\tau_{i n} \theta$. The corresponding abstract operation computes an abstract success substitution $\beta_{\text {out }}$ from $\beta_{i n}$. Correctness requires that $\tau_{\text {in }} \theta \in \gamma\left(\beta_{\text {out }}\right)$, i.e. any concrete computation which reaches the program point immediately after the built-in has an accumulated substitution whose projection upon the local environment of the clause is described by $\beta_{\text {out }}$.

Consider mode analysis where the abstract substitution gives for each local variable the mode which is one of free, ground and any. When unifying two variables say $X_{i}$ and $X_{j}$, it is not difficult to define an abstract operation which correctly computes the new modes for $X_{i}$ and $X_{j}$ from the old ones. However, for the other local variables, there is a problem. In case the mode of $X_{i}$ or $X_{j}$ has changed from free to any or ground, one has to change the mode of all free variables to any. Indeed, one cannot exclude that other variables share (have been unified) with one of $X_{i}$ or $X_{j}$. This is a very rough approximation. In order to obtain more precision, works on mode analysis include in one or another form a sharing component in the abstract substitutions.

Our novel approach consists of a propagation step which follows the abstract interpretation of the built-in. Only after this propagation step has updated the abstract substitution produced by the abstract interpretation of the built-in, do we require that $\tau_{i n} \theta$ is described by it. Consequently, the abstract interpretation of the built-in can be a much weaker (simpler) operation. Let us try to make our ideas more precise. One can say that the state reached by concrete execution is the result of a sequence of operations $O_{1}, \ldots, O_{n}$. Let $C S$ be the concrete state corresponding to it (e.g. the accumulated substitution). Performing another operation, say $O_{n+1}$, yields a new state $C S^{\prime}$. In the abstract interpretation framework, the part of $C S$ local to the clause on which $O_{n+1}$ is 
performed is abstracted as $A S$. With $A O_{n+1}$ the abstract counterpart of $O_{n+1}$, we have $A S^{\prime}=A O_{n+1}(A S)$ and correctness requires that $A S^{\prime}$ describes the local part of $C S^{\prime}$. For example, in Fig. $1 A S$ could be the abstract call substitution of $B_{j}$ and $A S^{\prime}$ its abstract success state (assuming $B_{j}$ is a call to a built-in).

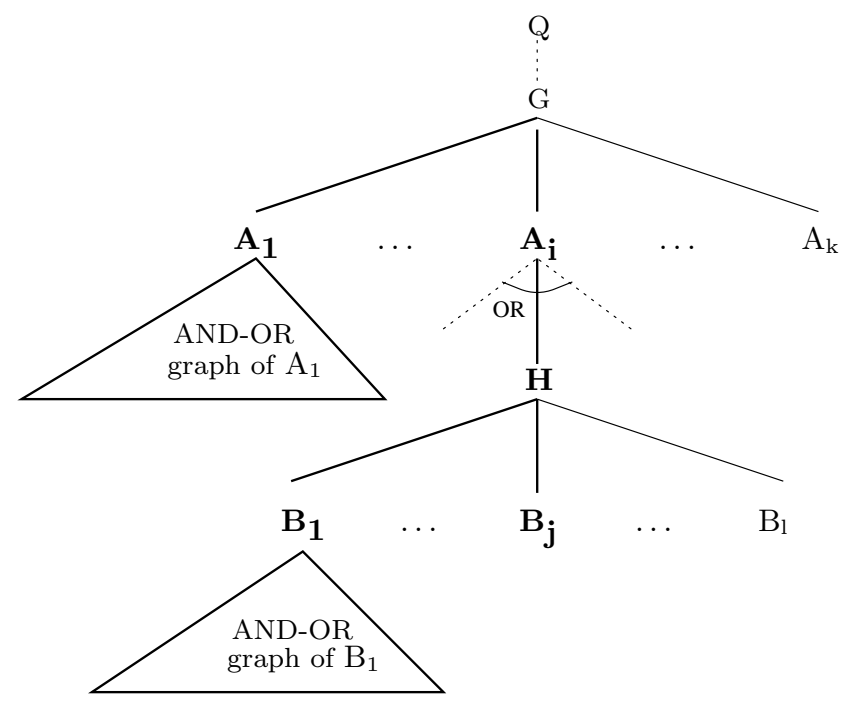

Fig. 1. Partial AND-OR-graph.

In the new framework, we assume that clauses are not only adorned with abstract call and success substitutions but also with a current state $A S_{c}$ which describes correctly the part of $C S$ local to the clause in question. In Fig. 1, assuming $B_{j}$ is to be executed, that means that all clauses on the path between $B_{j}$ and the root $\left(H \leftarrow B_{1}, \ldots, B_{l}, G \leftarrow A_{1}, \ldots, A_{k}, \ldots\right)$ are adorned with an $A S_{i}$ but also the clauses which can be involved in solving calls to the left of calls on the path to the root, i.e. clauses in the AND-OR subgraph for $B_{1}, \ldots$, in the ANDOR subgraph for $A_{1}, \ldots$. All these local abstract states $A S_{i}$ together make up a global state $A S$. In the new framework we have $A S^{\prime}=\operatorname{Prop}(A S)$ where both $A S^{\prime}$ and $A S$ are global states and Prop is a function which reexecutes all operations affecting $A S$ until a stable state is reached. In particular, if $O_{1}, \ldots, O_{n}, O_{n+1}$ is the sequence of operations leading to $C S^{\prime}$, then their abstract counterparts, say $A O_{1}, \ldots, A O_{n}, A O_{n+1}$, are reexecuted until a stable state is reached. All these operations $A O_{i}$ are weak operations, without propagation, which by themselves need not to be safe. The point is that when the global propagation ceases, a safe approximation is reached, i.e. all local states $A S_{i}^{\prime}$ correctly describe state $C S^{\prime}$ and this for any $C S$ represented by $A S$. The operations which need to be considered for reexecution are :

- All operations involved in reexecuting calls to the left of the path from the 
current program point to the root $\left(B_{1}, \ldots, B_{j-1}, A_{1}, \ldots, A_{i-1}, \ldots\right.$ in Fig. 1)

- The unifications between calls and headings on the path to the root (cfr. Fig. 1, unifications between $H$ and $A_{i}, G$ and ..., ...). Notice that the other ORbranches evolving from calls on the path to the root, are not reexecuted. (cfr. Fig. 1 : the other clauses defining $\left.A_{i}, \ldots\right)$.

At the level of implementation, the reexecution of abstract operations can be very selective. Only operations whose inputs have been modified need to be reexecuted. A good technique to locate such operations is to mark those variables in a call which are input to an operation which can affect the global state in the subgraph with the call as root (even better, to have pointers to the operation) and to mark those variables in the heading of a clause which are input to operations to be reached through the caller's environment. So when there is a change to the state of a local variable, it is easy to locate the abstract operations which can be affected by that change. Also caching techniques [8] can be used to speed up the reexecution. Finally notice that the global abstract state has to be adjusted when abstract interpretation of a particular clause is finished or temporarily interrupted and abstract interpretation of another clause is started/resumed. The new global abstract state can be restored from a table storing global states on context switching or computed through propagation starting from a known state : the latest abstract call/success substitution from all clauses contributing to the state.

Also procedure entry and procedure exit could be extended with a propagation step. With programs in normal form, procedure entry only copies the state of the variables in the call into the state of the variables in the head. So it is hard to imagine an application where propagation could be needed to obtain a safe procedure entry. Procedure exit copies information the other way around but also has to update the state of variables not participating in the call. Similar as for built-ins, it is feasible to have a weak procedure exit which needs to be followed by propagation to obtain a safe approximation.

As described in [1] the AND-OR tree is folded into a graph in case of recursion. E.g. in Fig. 2, the call $P\left(Y_{1}, \ldots, Y_{n}\right)$ refers back to its ancestor call $P\left(X_{1}, \ldots, X_{n}\right)$. We have that the projection of $\beta_{1}$ respectively $\beta_{2}$ upon $X_{1}, \ldots, X_{n}$ is equivalent to the projection upon $Y_{1}, \ldots, Y_{n}$ of $\beta_{3}$ respectively $\beta_{4}$. Propagation through recursion requires a special mentioning. Reexecuting $P\left(Y_{1}, \ldots, Y_{n}\right)$ means that one has to reexecute $P\left(X_{1}, \ldots, X_{n}\right)$. Reexecuting the unification between $P\left(X_{1}, \ldots, X_{n}\right)$ and $P\left(Z_{1}, \ldots, Z_{n}\right)$ means that one has also to update the abstract substitution in terms of $Y_{1}, \ldots, Y_{n}$.

\section{Mode Analysis}

We illustrate our novel framework with a mode analysis application. An abstract substitution over a set of variables $X_{1} \ldots X_{n}$ consists of a n-tuple of modes, the ith element being the mode for $X_{i}$. The possible modes are free, ground and any with their obvious meaning. No information about possible sharing is included. 


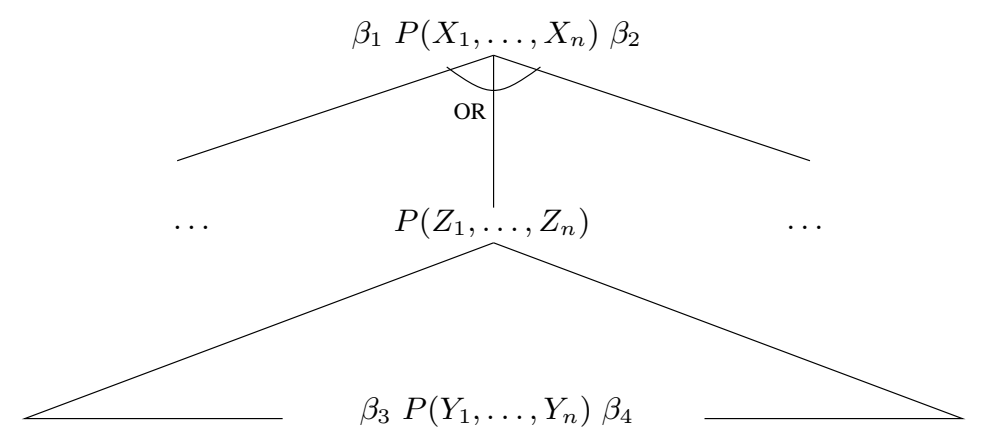

Fig. 2. AND-OR graph with a recursive call $P\left(Y_{1}, \ldots, Y_{n}\right)$.

Within existing frameworks, such an abstraction leads to very imprecise approximations. There is a very simple partial order over the modes of one variable free $<$ any and ground $<$ any. This partial order can easily be extended to n-tuples of modes : $\left(m_{1}, \ldots, m_{n}\right) \leq\left(m_{1}^{\prime}, \ldots, m_{n}^{\prime}\right)$ iff $m_{1} \leq m_{1}^{\prime}$ and $\ldots$ and $m_{n} \leq m_{n}^{\prime}$. We obviously have an abstract domain satisfying all requirements in [1]. Assuming that the top level query consists of a single call $Q / \mathrm{n}$, the abstract AND-OR graph is initialised with a root consisting of the call $Q\left(X_{1}, \ldots, X_{n}\right)$ and an abstract call substitution $\left(m_{1}, \ldots, m_{n}\right)$ specifying the mode of the class of queries being analysed. It is assumed that there is no sharing between the arguments of the concrete query. If sharing is desired, it has to be created explicitly in the code for $Q$.

Procedure entry (Fig. 3), an operation applied on a call $P\left(Y_{i_{1}}, \ldots, Y_{i_{m}}\right)$, performs abstract unification between the call and the heads of the defining procedures. With $P\left(X_{1}, \ldots, X_{m}\right)$ such a head, the effect is to pass the mode of $Y_{i_{j}}$ to $X_{j}$. In addition, the modes of the variables $X_{m+1}, \ldots, X_{n}$ local to the body of the defining procedure are initialised as free. Proving that this operation (without propagation) satisfies the condition imposed by the framework of [1] is straightforward.

Procedure exit (Fig. 3) considers the defining clauses one by one. Assume $P\left(X_{1}, \ldots X_{m}\right)$ is the head of a defining clause and $\left(m_{1}, \ldots, m_{m}, \ldots, m_{n}\right)$ the final abstract success substitution of the body. Assume $P\left(Y_{i_{1}}, \ldots Y_{i_{m}}\right)$ is the call. The modes $m_{1}, \ldots, m_{m}$ are passed on to the variables $Y_{i_{1}}, \ldots Y_{i_{m}}$. This yields a tuple $\left(m_{1}, \ldots m_{m}\right)$ of modes. Having such a tuple for every defining clause, the least upper bound is taken. Assuming that the call is part of a clause with variables $Y_{1}, \ldots, Y_{n}$, the variables in $\left\{Y_{1}, \ldots, Y_{n}\right\} \backslash\left\{Y_{i_{1}}, \ldots, Y_{i_{m}}\right\}$ are given the mode they had in the abstract call substitution for the call $P\left(Y_{i_{1}}, \ldots, Y_{i_{m}}\right)$. It is easy to prove that the modes for the variables $Y_{i_{j}}$ satisfy the condition of [1], however, the modes of the other variables do not satisfy the condition because the effects of sharing of variables from $\left\{Y_{1}, \ldots, Y_{n}\right\} \backslash\left\{Y_{i_{1}}, \ldots, Y_{i_{m}}\right\}$ and from $\left\{Y_{i_{1}}, \ldots, Y_{i_{m}}\right\}$ are ignored. It is claimed that the condition is satisfied after propagation. Propagation, as explained in Sect. 2, consists of :

- reexecuting (without propagations) calls to the left of the path from the 


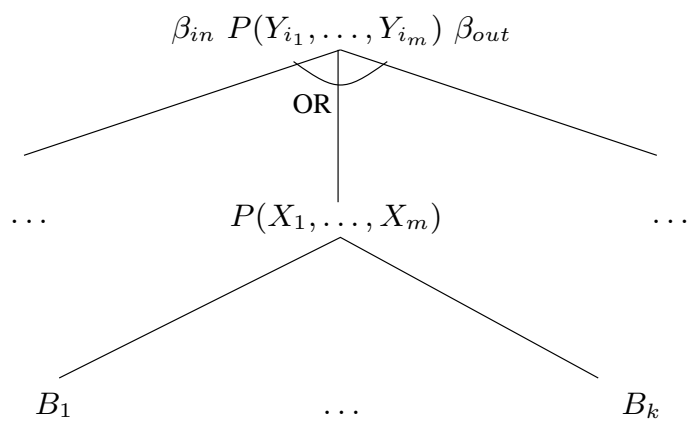

Fig. 3. Fragment of AND-OR graph with call $P\left(Y_{i_{1}}, \ldots, Y_{i_{m}}\right)$ and clause $P\left(X_{1}, \ldots, X_{m}\right) \leftarrow B_{1}, \ldots, B_{k}$.

current program point the call $P\left(Y_{i_{1}}, \ldots Y_{i_{m}}\right)$ to the root.

- reexecuting the unifications between calls and heads on the path from the current program point to the root. This is slightly simpler than procedure entry. Unification of a call $P\left(U_{1}, \ldots, U_{n}\right)$ with a head $P\left(V_{1}, \ldots, V_{n}\right)$ updates the mode of $U_{i}$ and $V_{i}$ as described below for the abstract interpretation of $X_{i}=X_{j}$ (for $1 \leq i \leq n$ ). Currently we have no formal proof of the claim, we discuss the claim in more detail after describing abstract interpretation of built-ins.

This propagation continues until a stable state is reached.

Abstract interpretation of built-ins is concerned with the effect of unification. Let $X_{1}, \ldots X_{n}$ be the variables of the clause. The abstract interpretation of the built-in $X_{i}=X_{j}$ is defined as follows : if either $X_{i}$ or $X_{j}$ has mode ground, both variables get mode ground; else if $X_{i}$ and $X_{j}$ have mode free, the modes are unchanged; else mode any is given to $X_{i}$ and $X_{j}$. For a unification $X_{i}=f\left(X_{j_{1}}, \ldots, X_{j_{m}}\right)$ one has similar rules : if either $X_{i}$ has mode ground or all of $X_{j_{k}}$ have mode ground, then all of $X_{i}, X_{j_{1}}, \ldots, X_{j_{m}}$ are given mode ground; else if $X_{i}$ has mode free, then its mode is changed to any while the modes of the $X_{j_{k}}$ are unchanged; else all modes become any. Again it is obvious that the modes of $X_{i}, X_{j_{1}}, \ldots, X_{j_{m}}$ satisfy the conditions of [1], but that propagation is needed to obtain correct modes for the other variables.

Claim Abstract interpretation of built-ins terminates and yields an abstract success substitution which satisfies the condition of [1], i.e. the abstract success substitution describes all concrete substitutions which can be observed in this program point.

Although we do not have a formal proof, we can mention some crucial elements.

Lemma. Propagation terminates.

The proof is based on the observation that the changes to the global state are monotone with regard to the instantiation order free $<$ any $<$ ground. 
Conjecture. Let $A S$ be an abstract substitution over the variables $X_{1}, \ldots, X_{n}$. Let $U_{1}, \ldots, U_{m}$ be a set of unifications involving variables from $X_{1}, \ldots, X_{n}$. Let $\theta$ be the mgu of $U_{1}, \ldots, U_{m}$. Let $W A U_{i}(S)$ be the abstract substitution resulting from the weak (without propagation) abstract interpretation of $U_{i}$ with abstract call substitution $S$. If $\sigma \in \gamma(A S)$ then $\sigma \theta \in \gamma\left(A S^{\prime}\right)$ with $A S^{\prime}$ the abstract substitution obtained by applying $W A U_{1}, \ldots, W A U_{m}$ starting in the state $A S$ until no more changes are observed.

The conjecture claims that the abstract interpretation of the $U_{i}$ can be done in isolation, ignoring the interaction with the other unifications and that propagation will recover the effects of the interaction. It is interesting to point out an example operation not satisfying the conjecture. Consider as operations $U_{i}$ numerical constraints of the form $a_{1} X_{1}+\ldots+a_{n} X_{n}=b$. Consider an abstract operation assigning ground to the mode of $X_{i}$ only if $X_{1}, \ldots, X_{i-1}, X_{i+1}, \ldots, X_{n}$ have mode ground and assigning any to the mode of $X_{i}$ only if $X_{1}, \ldots, X_{i-1}, X_{i+1}, \ldots, X_{n}$ have mode ground or any and at least one of them has mode any. Consider $X+Y=5, X-Y=2$ with modes free for $X$ and $Y$. Abstract interpretation of the individual statements will not change the modes of $X$ and $Y$, so also propagation will not modify them. However, a constraint solver for linear equations will, given the two equations, ground $X$ and $Y$. In other words the proposed abstraction (domain and operations) is not giving a correct approximation of the behaviour of the constraint solver.

\section{Some Examples}

We start with a simple artificial example :

$P(X, Y):-Q(X, Y), R(X), S(Y)$.

$Q(U, V):-U=V$.

$Q(U, V):-V=b$.

$R(U):-U=b$.

$S(U):-\ldots$

Assume the abstract state for the call to $P$ is $\{X=f, Y=f\}$. $Q$ has the same call state. Abstract interpretation of the first clause for $Q$ ends with a current state $\{U=f, V=f\}$; for the second clause, one obtains $\{U=f, V=g\}$. After taking the upper bound and renaming, one obtains $\{X=f, Y=a\}$ as abstract success state of $Q$ and both $X$ and $Y$ are marked as having non-local operations eligible for reexecution $(U=V$ in the first clause of $Q$ ). Because $V$ has mode ground in the local context of the second clause, and ground is the most instantiated state, one need not to add $V=b$ to the list of unifications to be redone upon modifying the mode of $Y$. We have applied this optimisation throughout our examples. After entering the clause defining $R$ (the variable $U$ in the head literal is marked as having a non-local unification), the unification $U=b$ is abstractly executed. It changes the mode of $U$ from $f$ to $g$. As there is a non-local operation, propagation is initiated. Back to the top level clause, the non-local operation on $X$ is found to be hidden in the call to $Q$. The execution of 
$Q$ is repeated, this time with abstract call state $\{X=g, Y=a\}$. Abstract interpretation of the first clause ends with $\{U=g, V=g\}$, for the second clause the result $\{U=g, V=g\}$ is already available, procedure exit yields $\{X=g, Y=g\}$ and one obtains the correct and precise result that $Y$ is ground when calling $S$ (also $X$ and $Y$ in $Q$ are no longer marked as having non-local operations). A simple mode analysis in a logic programming setting, keeping track of possible sharing will return from $Q$ with $\{X=f, Y=a\}$ and possible sharing between $X$ and $Y$. Executing $R$ will turn the mode of $X$ into $g$ but will leave $Y=a$ (if $Y$ were $f$ it would become $a$ due to the possible sharing with $X$ ) and one ends with the imprecise result that $Y$ has a mode $a$ when $S$ is called. It requires more sophisticated abstract domains such as the sharing domains in [5] and [11], the PROP domain of [10] and the EXP domain of [3] to obtain the more accurate result.

The next example involves recursion :

$Q(X, Y):-X=Y$.

$Q(X, Y):-X=f\left(X^{\prime}\right), Q\left(X^{\prime}, Y\right)$.

Consider the query ? $-Q(A, B), B=c$. with $\{A=f, B=f\}$. First, the call $Q(A, B)$ is executed. The first clause ends with state $\{X=f, Y=f\}$ while $X=Y$ is a candidate for reexecution (to reduce the amount of non-local propagation, such unifications between variables of the head could be returned as part of the abstract substitution). The second clause calls $Q$ recursively with the same pattern. Using the result of the first clause as an approximation, one obtains mode $a$ for $X, f$ for $Y$ and $X^{\prime}$ and a non-local operation on both $X^{\prime}$ and $Y$. Taking the upper bound of both clauses results in a new success state $\{X=a, Y=f\}$ with non-local operations on $X$ and $Y$, which is also the final one. Next, the call $B=c$ is considered : it grounds $B$ and triggers reexecution of $Q(A, B)$ with state $\{A=a, B=g\}$. The corresponding success state $\{A=g, B=g\}$ is computed by again considering the two clauses for $Q$ and using the result of the first one as an approximation for the recursive call. By reexecution $\{A=g, B=g\}$ is obtained as success state of the query.

Using an instantiation closed domain, e.g. modes $a$ and $g$, the propagation becomes optional. It was easy to adapt our existing abstract interpreter such that it only performed limited propagation : not doing propagation through the head into the caller's environment and only repeating the unifications amongst the previous calls in the body. We tested several programs. For a lot of simple programs, one obtains already good results as illustrated by Table 1 which contains for each program all the call states and the corresponding success states of the involved predicates. The code of the programs is found in Appendix A.

For other programs local propagation is not able to derive precise results. A good example is quicksort. $\operatorname{qsort}(L, S)$ :- $H=n i l, q s(L, S, H)$. 
Table 1. Call and success states of simple programs with limited local propagation.

\begin{tabular}{|l|ll|}
\hline \hline program & call state & success state \\
\hline \hline naive-reverse $(\mathrm{X}, \mathrm{Y})$ & $\{X=g, Y=a\}$ & $\{X=g, Y=g\}$ \\
append(Rt,H,Y) & $\{R t=g, H=g, Y=a\}$ & $\{R t=g, H=g, Y=g\}$ \\
\hline append(X,Y,Z) & $\{X=g, Y=g, Z=a\}$ & $\{X=g, Y=g, Z=g\}$ \\
\hline append(X,Y,Z) & $\{X=a, Y=a, Z=g\}$ & $\{X=g, Y=g, Z=g\}$ \\
\hline reverse $(\mathrm{X}, \mathrm{Y})$ & $\{X=g, Y=a\}$ & $\{X=g, Y=g\}$ \\
reverse(X,Y,Z) & $\{X=g, Y=a, Z=g\}$ & $\{X=g, Y=g, Z=g\}$ \\
\hline insert(E,OT,NT) & $\{E=g, O T=g, N T=a\}$ & $\{E=g, O T=g, N T=g\}$ \\
\hline insert(E,T) & $\{E=g, T=a\}$ & $\{E=g, T=a\}$ \\
\hline \hline
\end{tabular}

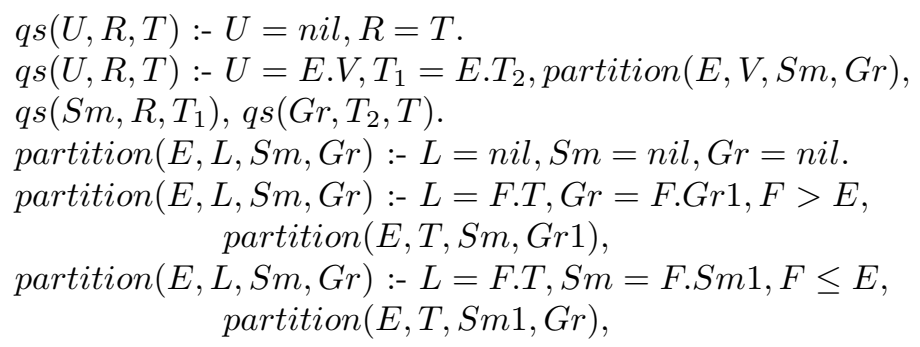

Abstract interpretation computes for the call state $\{X=g, Y=a\}$, the success state $\{X=g, Y=a\}$ with limited local propagation and is summarised by Table 2 which gives for each call state of qs $/ 3$ the call states of the recursive calls in the second clause and the success state.

Table 2. Call and success states for $\mathrm{qs} / 3$ with limited local propagation

\begin{tabular}{|l|l|l|}
\hline \hline Call state & $\begin{array}{l}\text { Call state 1st recursive call } \\
\text { Call state 2nd recursive call }\end{array}$ & Success state \\
\hline$\{U=g, R=a, T=g\}$ & $\left\{S m=g, R=a, T_{1}=a\right\}$ & $\{U=g, R=a, T=g\}$ \\
$\left\{U r=g, T_{2}=a, T=g\right\}$ & \\
$\{U=g, R=a, T=a\}$ & $\begin{array}{l}\left\{S m=g, R=a, T_{1}=a\right\} \\
\left\{G r=g, T_{2}=a, T=a\right\}\end{array}$ & $\{U=g, R=a, T=a\}$ \\
\hline \hline
\end{tabular}

Full propagation is needed to obtain the success state $\{X=g, Y=g\}$. For the sake of simplicity we use the modes $a$ and $g$, although including $f$ leads to similar results. With call state $S=\{U=g, R=a, T=g\}$ the abstract interpretation of the first clause computes a first approximation of the success state $\{U=g, R=g, T=g\}$. On procedure entry for the 2nd clause with call state $S$, the unification $T_{1}=E \cdot T_{2}$ remains eligible for reexecution and $T_{1}$ and $T_{2}$ have mode $a$. The abstract interpretation of the first recursive call marks the variables $R$ and $T_{1}$ - originating from the unification $R=T$ with $R$ and $T$ 
having mode $a$ in the first clause. The second recursive call uses the approximation and grounds $T_{2}$. Then $T_{1}=E \cdot T_{2}$ is reexecuted and grounds $T_{1}$. This forces the reexecution of the first recursive call as it is marked on $T_{1}$ with call state $\left\{S_{m}=g, R=a, T_{1}=g\right\}$. This call state is identical to the call state of its ancestor.

Finally, the abstract interpretation of a PROLOG III program [2] is worked out : mode analysis with modes $f, g$ and $a$ of the following lengthlist program which has in addition to unifications (represented as constraints) some typical numeric constraints :

lengthlist $(L, N):-\{L=\operatorname{nil}, N=0\}$.

lengthlist $(L, N):-\{L=E . T, N>0, N=N t+1\}$ lengthlist $(T, N t)$.

and the query? - lengthlist $(X, Y)$ with call state $S_{0}=\{X=g, Y=f\}$ (see Fig. 4). Constraint propagation has to deal with two typical numeric constraints.

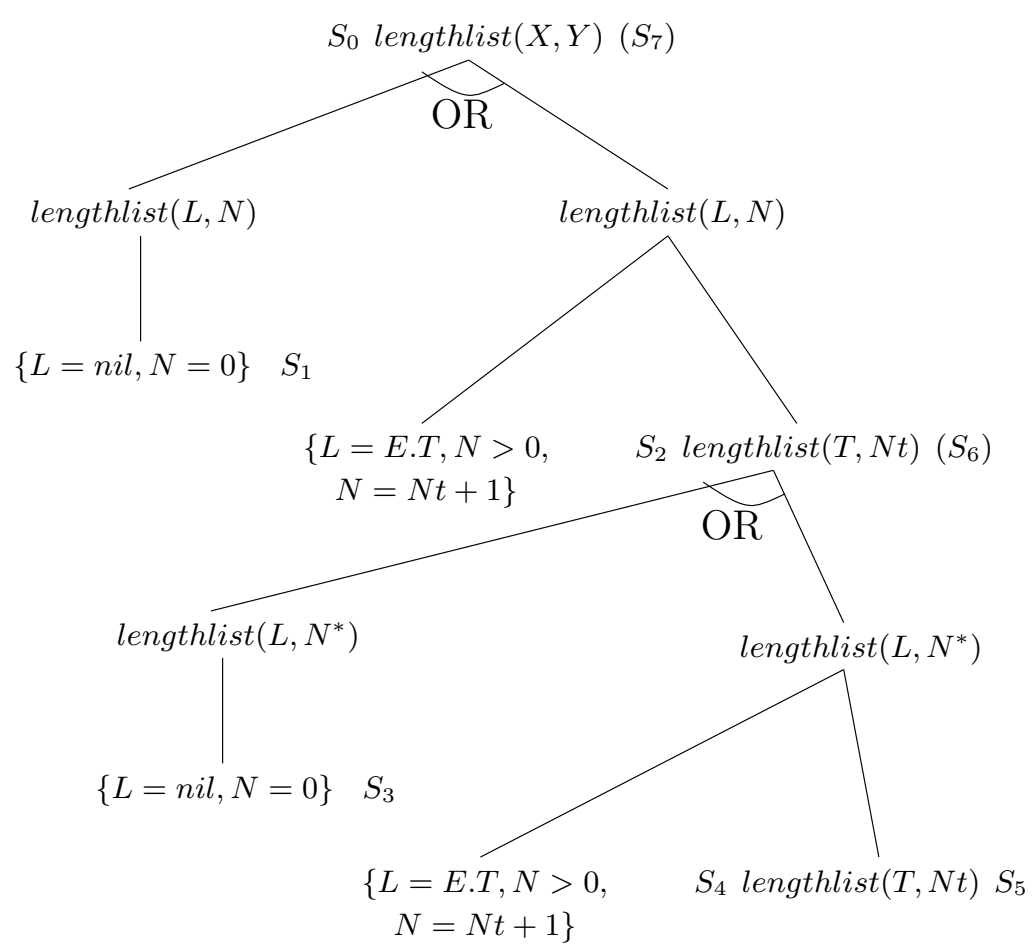

Fig. 4.

AND-OR graph for the call lengthlist $(X, Y)$ with call state $S_{0}=\{X=g, Y=f\}$ at the moment when propagation is started for $\mathrm{N}^{*}$.

The constraint $N>0$ changes the mode of $N$ from free to any (there could be another constraint $N \leq 0$ ) but it does not need to be reexecuted. For $N=N t+1$ 
there are two possible cases :

1. If $\mathrm{N}$ or $\mathrm{Nt}$ has mode $g$, then abstract interpretation turns both into $g$.

2. Otherwise, $N$ and $N t$ are given mode any while the constraint is eligible for reexecution.

Note that if both $N$ and $N t$ have mode $f$ before constraint propagation, constraint propagation imposes mode $a$ to them because they are possibly involved in more constraints : e.g. $\{N+N t=7, N-N t=3\}$ has one solution in which $\mathrm{N}$ and $\mathrm{Nt}$ are ground.

The abstract interpretation starts with call state $S_{0}$. Procedure entry computes $S_{1}=\{L=g, N=g\}$ and (after interpreting the constraints) $S_{2}=\{L=$ $g, N=a, E=g, T=g, N t=a\}$ with $N=N t+1$ eligible for reexecution. The projection of $S_{2}$ on the recursive call lengthlist $(T, N t)$ gives rise to a second call state $S^{\prime}=\{T=g, N t=a\}$ for lengthlist/2. So, procedure entry extends the AND-OR graph, marks the variable $N$ in the headings as having operations in the caller's environment eligible for reexecution and computes $S_{3}=\{L=g, N=g\}$ and $S_{4}=\{L=g, N=a, E=g, T=g, N t=a\}$. During the computation of $S_{3}$ the mode of the marked variable $N$ is changed. However propagation does not cause any changes (as $N$ is the only marked variable and it has mode ground, further instantiation is impossible and propagation can be avoided). In the second clause the projection of $S_{4}$ on lengthlist $(T, N t)$ is again $S^{\prime}$. An iterative process is started and the renaming of $S_{3}$ is used as a first approximation of the success state corresponding to $S^{\prime}$. Local propagation reactivates $N=N t+1$ $\left(N\right.$ gets mode $g$ ). This results in $S_{5}=\{L=g, N=g, E=g, T=g, N t=g\}$. Again the mode of the marked $N$ is changed. At this point we have computed the AND-OR graph of Fig. 4 and non-local propagation is triggered. State $S_{5}$, $\{T=g, N t=g\}$, is propagated into the caller's environment changing the mode of $N t$ from $a$ into $g$, the reexecution of $N=N t+1$ is triggered and $S_{2}$ is updated into $S_{2}^{\prime}=\{L=g, N=g, E=g, T=g, N t=g\}$. Now the recursive clause is reentered using procedure entry : $N$ is no longer marked, the updated state $S_{5}^{\prime}$ of the second clause is identical to $S_{5}$. In theory, $S_{5}^{c}$ should be obtained by computing $S_{4}^{c}=\{L=g, N=g, E=g, T=g, N t=g\}$ and computing the abstract interpretation of lengthlist $(T, N t)$ with call state $\{T=g, N t=g\}$, but the reexecution of lengthlist can again be avoided as it cannot further instantiate variables as they already have mode $g$ in $S_{5}$. Next, we continue with procedure exit for lengthlist : $S_{3}$ and $S_{5}^{\prime}$ are restricted and renamed into terms of $\{T, N t\}$; their upper bound $S^{\prime \prime}=\{T=g, N t=g\}$ yields the same result as the used approximation, so a fixed point has been computed. The next step in procedure exit is the extension which combines $S^{\prime \prime}$ with $S_{2}^{\prime}$. At this point the modes of $T$ and $N t$ remain unchanged, thus no propagation is triggered and $S_{6}=S_{2}^{\prime}$. Finally, procedure exit computes $S_{7}$ by combining $S_{0}$ ( which is still the current state for that program point) and the upper bound computed from $S_{1}$ and $S_{6}$ : $S_{7}=\{T=g, N t=g\}$. 


\section{Discussion}

We have sketched a novel framework for abstract interpretation of logic programming. We have illustrated the framework for the simple case of mode analysis of logic programs and have sketched how it can be extended to handle numerical constraints in a CLP language. With a very simple abstract domain, we obtain for a number of practical examples the same precision as other authors with substantially more complex domains as the sharing domain of [5], [11], the PROP domain of [10], the EXP domain of [3]. In our framework propagation is not only needed for precision, but also for correctness. Apparently we have traded simplicity of the framework against simplicity of the domain. Experimental evaluation will have to show whether our approach is advantageous. Compared to the repeat-previous-call strategy, whose practical value has been shown in [8], our propagation reactivates previous calls sooner. Reexecuting a call in the caller's environment is not delayed until normal procedure exit. Whether this has an advantageous effect upon the execution time of the analysis requires experimentation. Also more work has to be done in formalising our novel framework (including the handling of recursion) and the correctness of applications. A promising approach we are currently investigating is to reformulate the concepts in terms of the abstract OLDT framework [7]. Further work is required to find out whether other applications (in logic programming or constraint logic programming) can be simplified through the use of propagation in this novel framework. One candidate is the type analysis in [6] where the SVALand PSHR-components could be omitted substantially simplifying the abstract domain. However, that propagation terminates and yields safe approximations is less obvious than in the mode analysis case.

\section{Acknowledgement}

This work was funded in part by Diensten voor de Programmatie van het Wetenschapsbeleid (project RFO-AI-02) and ESPRIT project 5246 PRINCE. Maurice Bruynooghe is supported by the Belgian National Fund for Scientific Research. We are grateful to Danny De Schreye for reading the draft and discussing some technicalities and to Wim Simoens for the practical experiments.

\section{References}

1. M. Bruynooghe, "A Practical Framework for the Abstract Interpretation of Logic Programs", Journal of Logic Programming, Vol.10, pp. 91-124, (1991).

2. A. Colmerauer, "An Introduction to PROLOG III", Communications of the ACM, Vol.30(7), pp. 69-96 (1990).

3. A. Cortesi and G. File, "Abstract Interpretation of Logic Programs : an Abstract Domain for Groundness, Sharing, Freeness and Compoundness Analysis", Proc. Symposium on Partial Evaluation and Semantics-Based Program Manipulation, PEPMA'91, June 17-19, Connecticut, USA, pp. 52-61, (1991).

4. V. Dumortier, G. Janssens and M. Bruynooghe, "Detection of free variables in the presence of numeric constraints by means of abstract interpretation", $C W$ Report CW145, (1992). 
5. D. Jacobs and A. Langen, "Accurate and Efficient Approximation of Varaiable Aliasing in Logic Programs", "Proc. NACLP89, MIT-press, pp. 154-165, (1989).

6. G. Janssens and M. Bruynooghe, "Deriving Descriptions of Possible Values of Program Variables by Means of Abstract Interpretation : Definitions and Proofs", to appear in the Journal of Logic Programming 1992 (also published as CW Report CW107 Computer Science Dept., K.U.Leuven, March 1990)

7. T. Kanamori and T. Kawamura, "Abstract Interpretation Based on OLDT Resolution", to appear in the Journal of Logic Programming 1992

8. B. Le Charlier and P. Van Hentenryck, "Reexecution in Abstract Interpretation of Prolog", Technical Report No. CS-92-12, also to appear in Proc. JICLP92, (1992).

9. K. Marriott and H. Sondergaard, "Analysis of Constraint Logic Programs", Proc. NACLP90, ed. S. Debray and M. Hermenegildo, pp. 532-547, (1990).

10. K. Marriott and H. Sondergaard, "Notes for a Tutorial on Abstract Interpretation of Logic Programs", Proc. NACLP89, Cleveland, (1989).

11. K. Muthukamar and M. Hermenegildo, "Determination of Variable Dependence Information at Compile-Time through Abstract Interpretation", Pro. NACLP89, MIT-press, (1989).

\section{A Code of the Simple Example Programs in Table 1}

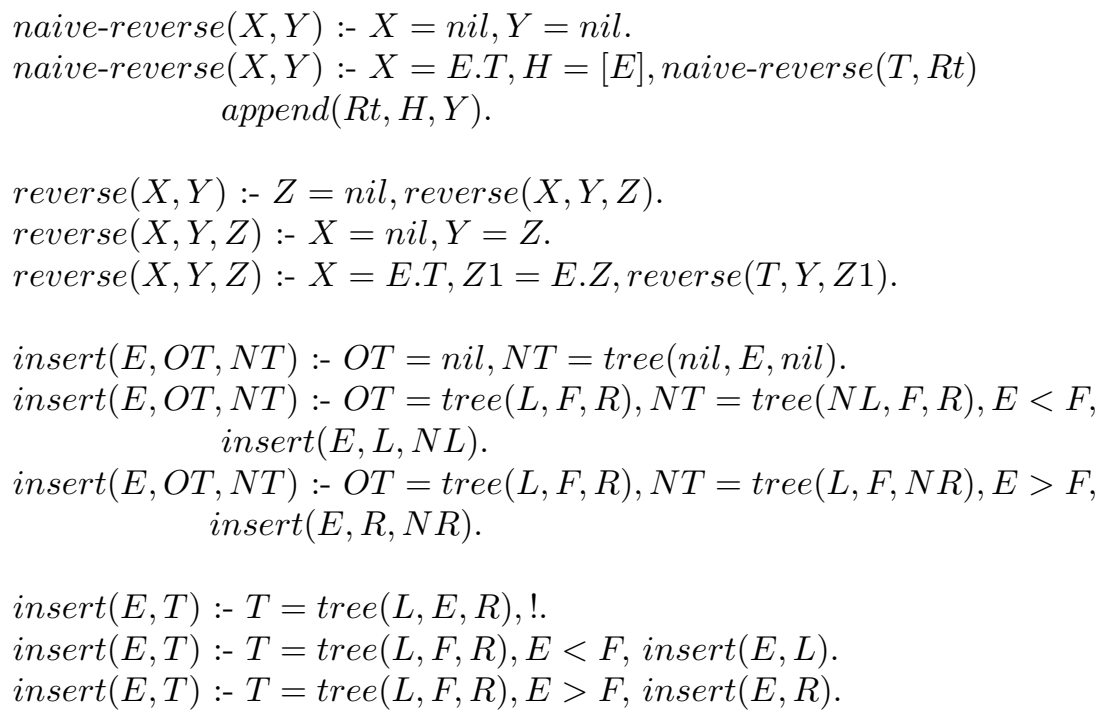

